\title{
SUPRIMENTO DO SILICATO DE CÁLCIO E A EFICIÊNCIA NUTRICIONAL DE VARIEDADES DE CAFEEIRO(1)
}

\author{
Adélia Aziz Alexandre Pozza ${ }^{(2)}$, Janice Guedes de Carvalho( ${ }^{(3)}$, Paulo \\ Tácito Gontijo Guimares ${ }^{(4)}$, Filipe Campos Figueiredo ${ }^{(5)} \&$ Alexandre \\ Romeiro Araújo ${ }^{(5)}$
}

\begin{abstract}
RESUMO
Embora não seja considerado nutriente essencial às plantas, o Si é classificado como elemento benéfico ou útil, mas pode alterar a dinâmica nutricional das plantas. Objetivando comparar a eficiência nutricional de variedades de cafeeiro (Catuaí, Mundo Novo e Icatu) em resposta à adubação silicatada, realizou-se um experimento em blocos casualizados, com as três variedades de mudas em tubetes combinadas com seis doses de $\mathrm{CaSiO}_{3}\left(\mathrm{~T}_{0}=0, \mathrm{~T}_{1}=0,063, \mathrm{~T}_{2}=0,125, \mathrm{~T}_{3}=0,25, \mathrm{~T}_{4}=0,5\right.$ e $\mathrm{T}_{5}=$ $1,0 \mathrm{~g} \mathrm{dm}^{-3}$ de substrato). A variedade Icatu teve a maior eficiência de absorção (EA) de Cu, Zn, Fe e Si, maior eficiência de uso (EU) de N, K, Ca, B e Mn, não diferindo da Mundo Novo com relação aos nutrientes N, Ca e Mn, e maior eficiência de translocação (ET) de N, S, Zn e de Fe. A Catuaí teve maior EA de P, K, B e Mn, não diferindo da Mundo Novo com relação aos nutrientes P, K e Mn, maior EU para Mg, $\mathrm{S}, \mathrm{Cu}, \mathrm{Zn}, \mathrm{Fe}$ e $\mathrm{Si}$, provavelmente devido à melhor ET desses nutrientes, exceto para Fe e Si. A Mundo Novo foi mais eficiente na absorção de N, K, Ca, Mg, Mn e Si, teve maior EU de P e Mn e maior ET de K, Ca, B, Mn e Si.
\end{abstract}

Termos de indexação: eficiência de translocação, absorção e uso, Coffea arabica, nutrição, adubação.

\footnotetext{
(1) Parte da Tese de Doutorado da primeira autora na Universidade Federal de Lavras - UFLA. Recebido para publicação em agosto de 2007 e aprovado agosto de 2009.

(2) Professora de Solos da Universidade Federal de Viçosa - UFV. Campus Florestal. CEP 35690-000 Florestal (MG). E-mail: adeliapozza@gmail.com

(3) Professora do Departamento de Ciência do Solo, UFLA. Bolsista do CNPq. E-mail: janicegc@ufla.br

(4) Pesquisador CTSM/EPAMIG, Caixa Postal 176, CEP 37200-000 Lavras (MG). E-mail: paulotgg@ufla.br

(5) Doutorando do Departamento de Ciência do Solo da Universidade Federal de Lavras - UFLA. Caixa Postal 3037, CEP 37200000 Lavras (MG). Bolsista da FAPEMIG. E-mails: adeliapozza@gmail.com; ffigueiredo@ufla.br; xande@navinet.com.br
} 


\title{
SUMMARY: EFFECT OF CALCIUM SILICATE SUPLLY AND THE NUTRITIONAL EFFICIENCY OF COFFEE CULTIVARS
}

\begin{abstract}
Silicon is classified as a beneficial nutrient and can improve the nutritional dynamics of plants, although it is not considered an essential nutrient for plants. In an experiment in a randomized block design, three coffee cultivars (Catuai, Mundo Novo and Icatu) were planted in plastic tubes, combined with six doses of calcium silicate $\left(T_{0}=0, T_{1}=0.063, T_{2}=0.125, T_{3}=0.25\right.$, $T_{4}=0.5$ and $T_{5}=1.0 \mathrm{~g} \mathrm{dm}^{-3}$ substrate), to compare the nutritional efficiency of the cultivars in response to silicon fertilization. Cultivar Icatu showed the higher uptake efficiency (UE) of Cu, $\mathrm{Zn}, \mathrm{Fe}$, and $\mathrm{Si}$, and efficiency of use (EU) of $N, K, C a, B$, and $M n$, but the later did not differ from Mundo Novo cultivar (N, Ca, and Mn). The efficiency of translocation (ET) was higher for $N, S, Z n$, and Fe. For Catuai the uptake efficiency of $P, K, B$, and Mn was higher, but did not differ from Mundo Novo for $P, K$, and $M n$. The Catuai's s efficiency of use for $M g, S, C u, Z n$, $F e$, and Si was higher, probably because of a better ET of these nutrients, with exception of Fe and Si. The cultivar Mundo Novo was the most efficient in N, K, Ca, Mg, Mn and Si uptake, had the higher EU of P and Mn, and the highest ET of K, Ca, B, Mn, and Si.
\end{abstract}

Index terms: translocation efficiency, uptake efficiency, use efficiency, Coffea arabica, nutrition, fertilization.

\section{INTRODUÇÃO}

Um dos grandes desafios da agricultura moderna é a utilização de estratégia de adaptação de plantas a solos de baixa fertilidade. Nesses casos, há duas alternativas: elevar a fertilidade dos solos pelo uso de corretivos e fertilizantes, ou empregar variedades nutricionalmente eficientes. O conceito de eficiência de plantas no uso de um nutriente engloba processos pelos quais elas absorvem, translocam, acumulam e utilizam melhor este nutriente para a produção de matéria seca ou grãos, em condições nutricionais normais ou adversas. A exigência nutricional e o crescimento das plantas variam de acordo com a espécie e o cultivar (Fageria, 1998; Martinez et al., 1993), dependendo da sua eficiência de absorção (Sands \& Mulligan, 1990; Duncan \& Baligar, 1990; Swiader et al., 1994), de translocação (Li et al., 1991; Duncan \& Baligar, 1990) e de uso dos nutrientes (Siddiqi \& Glass, 1981; Sands \& Mulligan, 1990; Duncan \& Baligar, 1990).

Vários mecanismos relacionados às características morfológicas e fisiológicas da planta contribuem para o uso eficiente de nutrientes, como: sistema radicular extensivo (que possibilita a exploração de maior volume de solo), alta relação entre raízes e parte aérea, habilidade do sistema radicular em modificar a rizosfera (possibilitando superar baixos teores de nutrientes), maior eficiência de absorção ou de uso de nutrientes, capacidade de manter o metabolismo normal com baixo teor de nutrientes nos tecidos e alta taxa fotossintética (Fageria \& Baligar, 1993).

A crescente demanda de nutrientes por variedades de café cada vez mais produtivas, assim como a expansão dessas lavouras para solos de baixa fertilidade, exige melhor compreensão da dinâmica dos nutrientes nessa cultura, evitando-se assim deficiência nutricional, altamente prejudicial aos programas de adubação (Reis Jr. \& Martinez, 2002).

Além dos elementos considerados essenciais para as plantas, há aqueles que são benéficos, como o $\mathrm{Si}$, favorecendo o crescimento e desenvolvimento normal das plantas (Malavolta, 2006; Korndörfer, 2006). A nutrição equilibrada com Si pode melhorar a arquitetura das plantas, reduzir a suscetibilidade ao estresse hídrico, aumentar a taxa fotossintética, além de alterar o $\mathrm{pH}$ da rizosfera das plantas. Mesmo sem possuir função físiológica e nutricional estabelecida, trabalhos indicam que o Si afeta o estado nutricional das culturas, como o cafeeiro (Santos Botelho et al., 2005) e o arroz (Ma \& Takahashi, 1990; Korndörfer \& Gascho, 1999). Dessa forma, acredita-se que o $\mathrm{Si}$ influencia a absorção de nutrientes e a eficiência nutricional das plantas.

Tendo em vista esses fatores, o objetivo deste trabalho foi comparar a eficiência de absorção, de translocação e de uso dos nutrientes N, P, K, Ca, Mg, $\mathrm{S}, \mathrm{B}, \mathrm{Cu}, \mathrm{Zn}, \mathrm{Fe}$ e Mn e do elemento Si entre variedades de cafeeiro e o efeito de doses de Si na eficiência nutricional dessas variedades.

\section{MATERIAL E MÉTODOS}

O experimento foi realizado em blocos casualizados, em esquema fatorial ( $3 \mathrm{X}$ 6), com três variedades (Catuaí Vermelho - IAC 99, Mundo Novo - IAC 379/ 19 e Icatu - IAC 2942) combinadas com seis doses de silicato de cálcio p.a. com $64 \%$ de $\mathrm{SiO}_{2}\left(\mathrm{~T}_{0}=0\right.$, $\mathrm{T}_{1}=0,063, \mathrm{~T}_{2}=0,125, \mathrm{~T}_{3}=0,25, \mathrm{~T}_{4}=0,5 \mathrm{e}$ $\mathrm{T}_{5}=1,0 \mathrm{~g} \mathrm{dm}^{-3}$ de substrato) e quatro repetições. A 
unidade experimental foi constituída por bandejas de tela com 16 tubetes de $120 \mathrm{~cm}^{3}$, totalizando 1.152 plantas, considerando-se como parcela útil (432 plantas) os seis tubetes centrais. O substrato foi constituído de $80 \%$ de esterco e $20 \%$ de terra de subsolo. Os tratamentos junto com o substrato foram colocados em saco plástico e agitados até homogeneizar a mistura. Retiraram-se amostras para análise química e da condutividade elétrica (Quadro 1).

Após o enchimento dos recipientes com os tratamentos e umedecimento do substrato por meio de irrigação, foi realizado o transplantio das três variedades de cafeeiro, no estádio de "palito de fósforo" produzidas em bandejas de areia lavada e esterilizada. A adubação foi realizada aplicando-se $0,75 \mathrm{~g}$ de fertilizante de liberação lenta (formulação 15-10-10 de $\mathrm{N}-\mathrm{P}-\mathrm{K}+$ micronutrientes) em cada tubete logo após o transplantio.

Aos sete meses de idade, as plantas foram colhidas, separadas em raízes e parte aérea, secas em estufa $\left(70{ }^{\circ} \mathrm{C}\right)$ até atingirem peso constante, quando se obteve a matéria seca de cada uma das partes. Os tecidos vegetais foram triturados em moinho tipo Wiley e as amostras destinadas às análises. O Si nos tecidos vegetais foi analisado segundo os métodos propostos por Gallo \& Furlani (1978). Para determinar os teores de N, P, K, Ca, Mg, S, B, Cu, Zn, Fe e Mn, seguiramse os métodos descritos por Malavolta et al. (1997). Calcularam-se as eficiências de absorção sugerida por Swiader et al. (1994) (nutriente total absorvido/ matéria seca da raiz), de translocação por $\mathrm{Li}$ et al. (1991) [(nutriente na parte aérea/nutriente na matéria seca total) x 100] e de uso por Siddiqi \& Glass (1981) (matéria seca total2/ nutriente na matéria seca total).

Foi realizada a análise de variância dos resultados. Em relação às doses de Si, foi empregada a análise de regressão para estimar a dose de Si que promoveu as melhores eficiências nutricionais. Realizou-se o teste de Scott \& Knott para comparar as variedades. O processamento dos dados foi feito utilizando-se o programa SISVAR. Escolheu-se o modelo de regressão que pudesse ser explicado biologicamente e testaramse os coeficientes das equações.

\section{RESULTADOS E DISCUSSÃO}

\section{Macronutrientes}

Observaram-se diferenças significativas entre as variedades quanto à produção de matéria seca e eficiência de uso, absorção e translocação de macronutrientes independente do tratamento (Quadro 2).

A maior produção de matéria seca (MS) foi observada na variedade Mundo Novo, conforme esperado, pois, geneticamente, esta variedade possui maior porte, folhas maiores e abundantes (Matiello et al., 2002) e, consequentemente, maior biomassa. O contrário foi observado na variedade Icatu, que apresentou as menores produções de matéria seca de raízes e também da parte aérea (Quadro 2).

A eficiência de uso é definida como a capacidade de utilização de nutrientes para síntese de MS (Souza, 1994). A eficiência de uso do nutriente absorvido pela planta é tão importante quanto a eficiência de absorção dos nutrientes, quando se avalia a produção de MS em relação ao suprimento de nutrientes. De acordo com os contrastes entre médias para eficiência de uso (EU), observou-se que a variedade Icatu sobressaiu com maior EU de $\mathrm{N}\left(0,59 \mathrm{~g}^{2} \mathrm{mg}^{-1}\right), \mathrm{K}\left(1,21 \mathrm{~g}^{2} \mathrm{mg}^{-1}\right) \mathrm{e}$ $\mathrm{Ca}\left(1,61 \mathrm{~g}^{2} \mathrm{mg}^{-1}\right)$, não diferindo da Mundo Novo para os nutrientes $\mathrm{N}\left(0,54 \mathrm{~g}^{2} \mathrm{mg}^{-1}\right)$ e $\mathrm{K}\left(1,14 \mathrm{~g}^{2} \mathrm{mg}^{-1}\right)$. Esses

Quadro 1. Atributos químicos e condutividade elétrica dos substratos constituídos por esterco de curral + terra de subsolo com as doses de silicato de cálcio $\left(T_{0}=0, T_{1}=0,063, T_{2}=0,125, T_{3}=0,25, T_{4}=0,5 e\right.$ $\mathrm{T}_{5}=1,0 \mathrm{~g} \mathrm{dm}^{-3}$ de substrato)

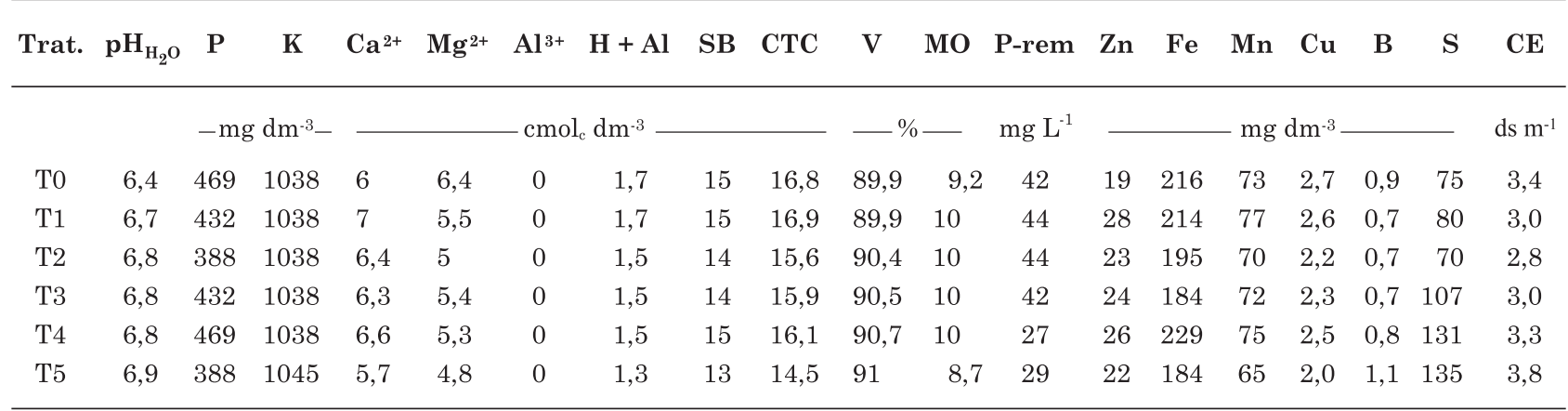

pH em água 1:2,5; P, Na, K, Fe, Mn e Cu: extrator Mehlich-1; Ca, Mg e Al: extrator KCl 1mol L'-1; H + Al: extrator acetato de cálcio 0,5 mol L-1: pH 7,0; B: extrator água quente; S: extrator fosfato monocálcico em ácido acético; SB: soma de bases trocáveis; CTC (t): capacidade de troca catiônica efetiva; CTC (T): capacidade de troca catiônica em pH 7,0; V: índice de saturação por bases; m: índice de saturação por Al; MO: matéria orgânica: carbono orgânico x 1,724: Walkley-Black; P-rem: fósforo remanescente; CE: condutividade elétrica. 
Quadro 2. Produção média de matéria seca da parte aérea (PA), raízes e total, e eficiência de uso (EU), absorção (EA) e translocação (ET) de macronutrientes por mudas de três variedades de cafeeiro produzidas em tubetes, independente do tratamento

\begin{tabular}{|c|c|c|c|c|}
\hline Característica & Catuaí & Mundo Novo & Icatu & CV (\%) \\
\hline Matéria seca da PA (g) & $1,50 \mathrm{~b}$ & $1,83 \mathrm{a}$ & $1,54 \mathrm{~b}$ & 15,00 \\
\hline Matéria seca de raízes (g) & $0,58 \mathrm{a}$ & $0,54 \mathrm{a}$ & $0,47 \mathrm{~b}$ & 18,64 \\
\hline Matéria seca total (g) & $2,08 \mathrm{~b}$ & $2,38 \mathrm{a}$ & $2,01 \mathrm{~b}$ & 14,51 \\
\hline EU de $N\left(g^{2} \mathrm{mg}^{-1}\right)$ & $0,44 \mathrm{~b}$ & $0,54 \mathrm{a}$ & $0,59 \mathrm{a}$ & 17,52 \\
\hline EA de $N\left(\mu \mathrm{g} \mathrm{g}^{-1}\right)$ & $0,173 \mathrm{~b}$ & $0,196 \mathrm{a}$ & $0,153 \mathrm{c}$ & 16,45 \\
\hline ET de N (\%) & $50,31 \mathrm{~b}$ & $50,96 \mathrm{~b}$ & $56,02 \mathrm{a}$ & 8,52 \\
\hline EU de $\mathrm{P}\left(\mathrm{g}^{2} \mathrm{mg}^{-1}\right)$ & $3,69 \mathrm{c}$ & $5,48 \mathrm{a}$ & $5,01 \mathrm{~b}$ & 14,59 \\
\hline EA de $\mathrm{P}\left(\mu \mathrm{g} \mathrm{g}^{-1}\right)$ & $0,020 \mathrm{a}$ & $0,019 \mathrm{a}$ & $0,017 \mathrm{~b}$ & 12,93 \\
\hline ET de P (\%) & $51,79 \mathrm{~b}$ & $61,45 \mathrm{a}$ & $52,65 \mathrm{~b}$ & 3,70 \\
\hline EU de $\mathrm{K}\left(\mathrm{g}^{2} \mathrm{mg}^{-1}\right)$ & $0,82 \mathrm{~b}$ & $1,14 \mathrm{a}$ & $1,21 \mathrm{a}$ & 17,83 \\
\hline EA de $\mathrm{K}\left(\mu \mathrm{g} \mathrm{g}^{-1}\right)$ & $0,092 \mathrm{a}$ & $0,093 \mathrm{a}$ & $0,073 \mathrm{~b}$ & 15,32 \\
\hline ET de K (\%) & $45,43 \mathrm{c}$ & $61,59 \mathrm{a}$ & $55,21 \mathrm{~b}$ & 8,30 \\
\hline EU de $\mathrm{Ca}\left(\mathrm{g}^{2} \mathrm{mg}{ }^{-1}\right)$ & $1,14 \mathrm{c}$ & $1,39 \mathrm{~b}$ & $1,61 \mathrm{a}$ & 16,67 \\
\hline $\mathrm{EA}$ de $\mathrm{Ca}\left(\mu \mathrm{g} \mathrm{g}^{-1}\right)$ & $0,066 \mathrm{~b}$ & $0,075 \mathrm{a}$ & $0,055 \mathrm{c}$ & 13,75 \\
\hline ET de $\mathrm{Ca}(\%)$ & $49,60 \mathrm{~b}$ & $54,73 \mathrm{a}$ & $47,98 \mathrm{~b}$ & 6,92 \\
\hline EU de $\mathrm{Mg}\left(\mathrm{g}^{2} \mathrm{mg}{ }^{-1}\right)$ & $4,07 \mathrm{a}$ & $3,20 \mathrm{~b}$ & $2,64 \mathrm{c}$ & 14,78 \\
\hline EA de $\mathrm{Mg}\left(\mu \mathrm{g} \mathrm{g}^{-1}\right)$ & $0,018 \mathrm{~b}$ & $0,033 \mathrm{a}$ & $0,033 \mathrm{a}$ & 13,16 \\
\hline ET de $\mathrm{Mg}(\%)$ & $52,46 \mathrm{a}$ & $34,99 \mathrm{c}$ & $48,29 \mathrm{~b}$ & 6,01 \\
\hline EU de $\mathrm{S}\left(\mathrm{g}^{2} \mathrm{mg}^{-1}\right)$ & 7,83 a & $4,86 \mathrm{~b}$ & $2,91 \mathrm{c}$ & 25,62 \\
\hline EA de $S\left(\mu \mathrm{g} \mathrm{g}^{-1}\right)$ & $0,010 \mathrm{c}$ & $0,022 \mathrm{~b}$ & $0,030 \mathrm{a}$ & 19,52 \\
\hline ET de S (\%) & $50,87 \mathrm{a}$ & $24,88 \mathrm{~b}$ & $50,44 \mathrm{a}$ & 13,65 \\
\hline
\end{tabular}

Médias seguidas de mesma letra na linha não diferiram entre si pelo teste de Scott \& Knott a $5 \%$. Todos os valores das características descritas foram significativos a $1 \%$, pelo teste $\mathrm{F}$.

resultados concordam com estudo realizado por Corrêa et al. (1983), em que a variedade Mundo Novo foi mais eficiente no uso do $\mathrm{N}$ do que a Catuaí. Quanto ao Ca, Tomaz et al. (2003) também obtiveram maior EU do Ca na variedade Mundo Novo $\left(1,01 \mathrm{~g}^{2} \mathrm{mg}^{-1}\right)$ em relação à Catuaí $\left(0,76 \mathrm{~g}^{2} \mathrm{mg}^{-1}\right)$, porém não observaram diferenças quanto à eficiência de translocação e de absorção deste nutriente entre as variedades estudadas.

A maior eficiência de uso de $\mathrm{N}$ pode ser atribuída à maior produção de MS pela parte aérea da variedade Mundo Novo. Vários estudos em outras culturas mostram o comportamento diferencial entre espécies ou variedades da mesma espécie na absorção e no uso de N (Wuest \& Cassman, 1992; Fageria \& Baligar, 1993). Furlani et al. (1986), avaliando o comportamento diferencial de linhagens de arroz na absorção e no uso de $\mathrm{N}$ em solução nutritiva, verificaram que a diferenciação entre as plantas deveu-se principalmente à capacidade de uso do N, promovendo a variação nos pesos de MS total.

A eficiência de uso do $\mathrm{P}$ foi maior na variedade Mundo Novo, isto é, produziu em média 0,55 g de MS por $1 \mathrm{mg}$ de $\mathrm{P}$ absorvido, enquanto as variedades Icatu e Catuaí produziram 0,50 e 0,37 $\mathrm{g} \mathrm{mg}^{-1}$. A maior eficiência de uso do $\mathrm{P}$ pode ser um fator de grande importância em condições limitantes deste mineral, pois a planta poderia produzir mais com menor exigência deste nutriente. Para Gerloff \& Galbelman (1983), a maior eficiência de uso pode ser devido à menor necessidade de $\mathrm{P}$ para reações bioquímicas da planta, à maior redistribuição do nutriente para os pontos de crescimento e à maior mobilização do $\mathrm{P}$ armazenado nos vacúolos das células. Abichequer \& Bohnen (1998), avaliando a eficiência nutricional de variedades de trigo quanto ao $\mathrm{P}$, demonstraram que as variedades eficientes e ineficientes no aproveitamento do $\mathrm{P}$ da solução nutritiva diferenciaram-se quanto à capacidade de translocar o $\mathrm{P}$ para a parte aérea e utilizá-lo na produção de matéria seca. Whiteaker et al. (1976), em estudo com linhagens de feijão mais eficientes no uso do $\mathrm{P}$, observaram que, nos tratamentos com baixas doses de $\mathrm{P}$, as plantas das linhagens com maior EU, apresentaram maiores taxas fotossintéticas. Com isso, associou-se a EU de $\mathrm{P}$ ao aumento do metabolismo fotossintético.

A variedade Catuaí teve maior EU para o $\mathrm{Mg}$ e o $\mathrm{S}$, provavelmente devido à melhor eficiência de translocação desses nutrientes. Entretanto, Tomaz et al. (2003), avaliando a eficiência nutricional de Ca 
e de Mg em mudas de Coffea arabica e C. canephora, não observaram diferença quanto à ET, e as variedades Catuaí 15 e Conilon M.1 tiveram os menores valores de EU de Mg. Quanto ao S, Souza (1999) atribuiu a maior EU do S do cultivar Rubi ao maior sistema radicular, relacionada com a maior aquisição do nutriente no solo, tendo o mesmo sido verificado neste experimento para a variedade Catuaí $(0,58 \mathrm{~g}$ de raízes secas). Essa variedade, segundo Matiello et al. (2002), possui amplo sistema radicular, muitas vezes superior ao do Mundo Novo.

Variedades de muitas espécies de plantas apresentam diferenças na absorção de nutrientes. As diferenças entre as plantas quanto à capacidade de absorver nutrientes são decorrentes tanto da variação na magnitude e morfologia do sistema radicular quanto da cinética de absorção de nutrientes. Variações nos parâmetros morfológicos de raiz (sistema radicular extensivo e eficiente) e nos cinéticos de absorção (Vmáx, Km e Cmín) são necessárias para operar os modelos que descrevem quantitativamente o processo de absorção dos nutrientes (Anghinoni et al., 1989).

Para a eficiência de absorção (EA), a variedade Mundo Novo sobressaiu com maior EA de N, P, K, Ca e Mg, não diferindo da Catuaí com os nutrientes $\mathrm{P}$ e $\mathrm{K}$ e nem da Icatu com o Mg. Já a variedade Icatu absorveu mais $\mathrm{S}$ do que as outras. A variedade mais vigorosa e, portanto, com perspectivas de maior produção, possui um sistema radicular mais extenso, explora maior volume de solo e intercepta maior quantidade de nutrientes, facilitando a absorção. Comparando a variedade Catuaí com a Conilon, Reis Jr. \& Martinez (2002) observaram maior EA de P para a Catuaí $\left(0,0075 \mu \mathrm{g} \mathrm{g}^{-1}\right.$ de $\mathrm{P}$ na MS raiz), sendo este valor mais baixo do que o encontrado neste trabalho $\left(0,020 \mu \mathrm{g} \mathrm{g}^{-1} \mathrm{de} \mathrm{P}\right.$ na MS raiz). A variedade mais eficiente na translocação do $\mathrm{N}$ e do $\mathrm{S}$ foi a Icatu, não diferindo da Catuaí para o S. A variedade Mundo Novo destacou-se na translocação de P, K e Ca, sendo a Catuaí mais eficiente na translocação de $\mathrm{Mg}$ e S. Um possível fator determinante dessa maior EA e da translocação do S pela variedade Icatu seria a sua capacidade de redistribuir as frações solúvel e insolúvel de $\mathrm{S}$ das folhas maduras para as folhas novas, dependendo do estado nutricional da planta (Sunarpi Anderson, 1996).

A Mundo Novo translocou (ET) proporcionalmente mais $\mathrm{P}$ para a parte aérea do que a Catuaí e a Icatu, isto é, para cada $1 \mathrm{mg}$ de $\mathrm{P}$ absorvido, 0,615 $\mathrm{mg}$ foi transportado para a parte aérea dessa variedade (Quadro 2). Observou-se também diferença significativa para ET de P nessa mesma variedade com as doses de Si (Figura 1a), entretanto nenhum dos modelos testados apresentou ajuste aos dados. Observou-se redução na ET com o aumento das doses de silicato somente até $0,25 \mathrm{~g} \mathrm{dm}^{-3}$; após esse valor houve novo aumento. Em arroz, Méndez Baldeon (1995) testou o uso do termofosfato magnesiano, do superfosfato triplo e do superfosfato triplo mais calagem. O efeito favorável do termofosfato sobre o aproveitamento do $\mathrm{P}$ foi devido, provavelmente, à sua capacidade de elevar o $\mathrm{pH}$ do meio e não devido à competição entre o Si do termofosfato e o $\mathrm{P}$ pelos mesmos sítios de adsorção no solo. Nesse experimento, a competição do fosfato com o silicato reduziu a eficiência de translocação do $\mathrm{P}$ para a variedade Mundo Novo.

Independentemente da variedade, houve diferença $(\mathrm{P}=0,05)$ entre as doses de Si para ET de K, (Figura 1b). Para as variáveis ET de $\mathrm{P}$ da variedade Icatu (Figura 1c) e EU do Mg da Mundo Novo (Figura 1d), observaram-se diferenças significativas a $1 \%$ pelo teste de Scott \& Knott, para ambas. Embora os modelos de regressão testados não tenham se ajustado aos dados para explicar esses fenômenos,
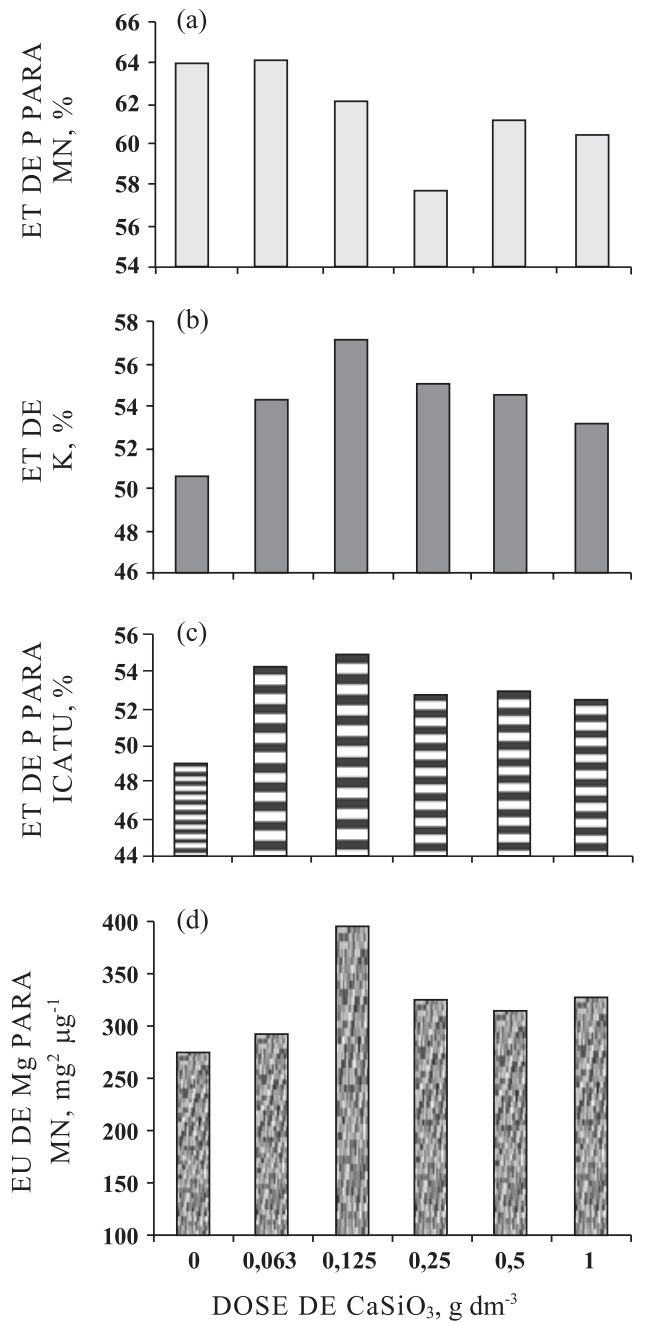

Figura 1. Eficiência de translocação (ET) de $P$ para a variedade Mundo Novo (MN) (a), ET média de K no cafeeiro (b), ET de P para Icatu (c) e eficiência de uso (EU) do Mg para a variedade Mundo Novo (MN) (d), em função das doses de silicato de cálcio $\left(\mathrm{CaSiO}_{3}\right)$ aplicadas ao substrato de plantio. 
observou-se a diferença entre a testemunha sem Si e as outras doses. Ocorreu aumento da eficiência nutricional com o aumento das doses de Si em relação à dose zero de $\mathrm{CaSiO}_{3}$. Houve aumento de $12 \%$ na ET de P para a variedade Icatu (Figura 1c), $13 \%$ na ET de $\mathrm{K}$, independentemente da variedade (Figura $2 \mathrm{~b}$ ) e $43 \%$ de aumento da EU de Mg pela variedade Mundo Novo para a dose $0,125 \mathrm{~g}$ de $\mathrm{CaSiO}_{3} \mathrm{dm}^{-3}$ de substrato (Figura 1d).

\section{Micronutrientes}

Todas as variedades diferiram entre si a $1 \%$ de probabilidade pelo teste $\mathrm{F}$, e as médias foram comparadas pelo teste de Scott \& Knott a $5 \%$ de probabilidade (Quadro 3). A variedade Catuaí teve maior eficiência de uso dos micronutrientes $\mathrm{Cu}, \mathrm{Zn}$ e $\mathrm{Fe}$ e também de Si. A Icatu, por sua vez, teve maior eficiência de uso do B e do Mn.

Estudo com adição de Zn e absorção, translocação e uso de Zn e P por variedades de cafeeiro indicou o Conilon como mais eficiente no uso de Zn comparado ao Catuaí, isto é, o Conilon produziu, em média, $0,15 \mu \mathrm{g} \mathrm{g}^{-1}$ de MS para o $\mathrm{Zn}$ absorvido, enquanto o Catuaí produziu $0,11 \mu \mathrm{g} \mathrm{g}^{-1}$ de $\mathrm{MS}$ de $\mathrm{Zn}$ absorvido (Reis Jr. \& Martinez, 2002). Neste experimento, Catuaí $\left(73,96 \mathrm{mg}^{2} \mu \mathrm{g}^{-1}\right)$ foi superior às variedades Mundo Novo e Icatu (20,31 e 8,15 mg ${ }^{2} \mu \mathrm{g}^{-1}$, respectivamente), porém teve menor EU, se comparada à EU de Zn obtida por Reis Jr. \& Martinez (2002). Quanto à EA, por outro lado, a variedade Icatu foi mais eficiente na absorção do $\mathrm{Cu}$, do $\mathrm{Zn}$, do Fe e do Si, enquanto a Catuaí absorveu mais B e Mn. Da mesma forma, Reis Jr. \& Martinez (2002) encontraram maior eficiência de absorção de $\mathrm{Zn}$ para Catuaí $\left(52,5 \mu \mathrm{g} \mathrm{g}^{-1}\right)$ do que para Conilon $\left(29,8 \mu \mathrm{g} \mathrm{g}^{-1}\right)$.

Quanto à eficiência de translocação, a variedade Catuaí translocou mais $\mathrm{Cu}$ e Zn, a Mundo Novo o B, $\mathrm{Mn}$ e Si, e a Icatu translocou mais Fe do que as outras e comportou-se de modo semelhante ao da Catuaí para o Zn (Quadro 3). Não se observaram diferenças significativas das doses de $\mathrm{Si}$ sobre a eficiência nutricional das variedades quanto a $\mathrm{EU}$ de $\mathrm{B}, \mathrm{Cu}, \mathrm{Zn}$, Fe e Mn, EA de B e Mn, ET de B, Cu e Fe.

Observou-se diferença significativa para eficiência de absorção de Cu nas mudas da variedade Icatu com as doses de $\mathrm{Si}$, tendo havido redução até a dose 0,48 $\mathrm{g} \mathrm{dm}^{-3}$ de $\mathrm{CaSiO}_{3}$ (Figura 2a).

O aumento das doses de Si reduziu EA de cobre também para a variedade Mundo Novo (Figura 2b), exceto para a dose $0,25 \mathrm{~g} \mathrm{dm}^{-3} \mathrm{de}^{\mathrm{CaSiO}} 3$. Nessa dose, a EA de $\mathrm{Cu}$ foi $18 \%$ maior do que na testemunha e, para as outras doses, foi menor. Observou-se diferença significativa para a EA de Zn pelas mudas da variedade Icatu com as doses de Si. A dose zero de Si, ou seja, a testemunha, comportou-se semelhantemente às plantas adubadas com a dose $0,5 \mathrm{~g} \mathrm{dm}^{-3}$ e foi inferior

Quadro 3. Produção média de matéria seca da parte aérea, das raízes e total, e eficiência de uso (EU), absorção (EA) e translocação (ET) de micronutrientes e do silício por mudas de três variedades de cafeeiro produzidas em tubetes, independente dos tratamentos

\begin{tabular}{|c|c|c|c|c|}
\hline Parâmetro & Catuaí & Mundo Novo & Icatu & CV (\%) \\
\hline EU de B $\left(\mathrm{mg}^{2} \mu \mathrm{g}^{-1}\right)$ & $24,21 \mathrm{c}$ & $37,08 \mathrm{~b}$ & $45,49 \mathrm{a}$ & 18,66 \\
\hline EA de B $\left(\mu g g^{-1}\right)$ & $0,311 \mathrm{a}$ & $0,287 \mathrm{~b}$ & $0,198 \mathrm{c}$ & 15,49 \\
\hline ET de B $(\%)$ & $51,39 \mathrm{~b}$ & $62,10 \mathrm{a}$ & $52,87 \mathrm{~b}$ & 7,09 \\
\hline $\mathrm{EU}$ de $\mathrm{Cu}\left(\mathrm{mg}^{2} \mu \mathrm{g}^{-1}\right)$ & $404,16 \mathrm{a}$ & $197,34 \mathrm{~b}$ & $118,51 \mathrm{c}$ & 22,73 \\
\hline $\mathrm{EA}$ de $\mathrm{Cu}\left(\mu \mathrm{g} \mathrm{g}^{-1}\right)$ & $0,0192 \mathrm{c}$ & $0,0568 \mathrm{~b}$ & $0,075 \mathrm{a}$ & 19,25 \\
\hline $\mathrm{ET}$ de $\mathrm{Cu}(\%)$ & $57,89 \mathrm{a}$ & $19,34 \mathrm{c}$ & $52,89 \mathrm{~b}$ & 16,67 \\
\hline EU de $\mathrm{Zn}\left(\mathrm{mg}^{2} \mu \mathrm{g}^{-1}\right)$ & $73,96 \mathrm{a}$ & $20,31 \mathrm{~b}$ & $8,15 \mathrm{c}$ & 30,07 \\
\hline EA de $\mathrm{Zn}\left(\mu \mathrm{g} \mathrm{g}^{-1}\right)$ & $0,105 \mathrm{c}$ & $0,537 \mathrm{~b}$ & $1,10 \mathrm{a}$ & 18,71 \\
\hline ET de Zn (\%) & $49,06 \mathrm{a}$ & $11,40 \mathrm{~b}$ & $50,56 \mathrm{a}$ & 13,99 \\
\hline EU de $\mathrm{Fe}\left(\mathrm{mg}^{2} \mu \mathrm{g}^{-1}\right)$ & $1,50 \mathrm{a}$ & $1,21 \mathrm{~b}$ & $0,88 \mathrm{c}$ & 18,91 \\
\hline EA de $\mathrm{Fe}\left(\mu \mathrm{g} \mathrm{g}^{-1}\right)$ & $5,09 \mathrm{c}$ & $8,86 \mathrm{~b}$ & $10,47 \mathrm{a}$ & 19,52 \\
\hline ET de Fe (\%) & $47,84 \mathrm{~b}$ & $45,87 \mathrm{~b}$ & $60,19 \mathrm{a}$ & 12,13 \\
\hline EU de $\mathrm{Mn}\left(\mathrm{mg}^{2} \mu \mathrm{g}^{-1}\right)$ & $9,96 \mathrm{~b}$ & $14,34 \mathrm{a}$ & $15,29 \mathrm{a}$ & 18,86 \\
\hline EA de $\mathrm{Mn}\left(\mu \mathrm{g} \mathrm{g}^{-1}\right)$ & $0,77 \mathrm{a}$ & $0,75 \mathrm{a}$ & $0,59 \mathrm{~b}$ & 19,60 \\
\hline ET de Mn (\%) & $52,08 \mathrm{~b}$ & 70,15 a & $54,79 \mathrm{~b}$ & 11,15 \\
\hline EU de $\mathrm{Si}\left(\mathrm{g}^{2} \mathrm{mg}^{-1}\right)$ & $460,74 \mathrm{a}$ & $255,65 \mathrm{~b}$ & $224,87 \mathrm{~b}$ & 25,64 \\
\hline EA de $\mathrm{Si}\left(\mathrm{mg} \mathrm{g}^{-1}\right)$ & $0,017 \mathrm{~b}$ & $0,046 \mathrm{a}$ & $0,042 \mathrm{a}$ & 30,63 \\
\hline ET de Si (\%) & $54,33 \mathrm{~b}$ & $65,74 \mathrm{a}$ & $44,96 \mathrm{c}$ & 13,67 \\
\hline
\end{tabular}

Médias seguidas de mesma letra na linha não diferiram entre si por Scott \& Knott a 5 \%. Todos os valores das características avaliadas foram significativos, a $1 \%$, pelo teste $\mathrm{F}$. 
às demais doses (Figura 2c). A cultura do cafeeiro é exigente em micronutrientes, especialmente o Zn. Esse micronutriente tem comprometido a produtividade da
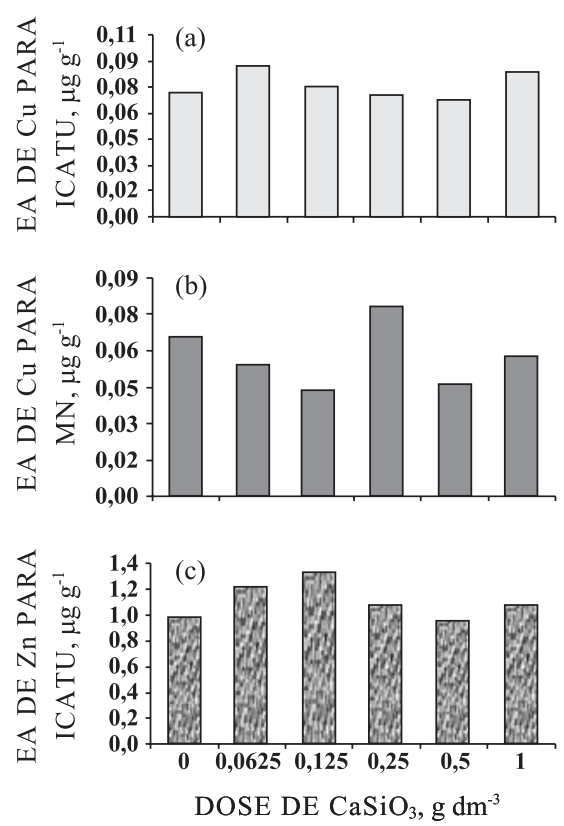

Figura 2. Eficiência de absorção (EA) de cobre pelas variedades Icatu (a), Mundo Novo (b) e de zinco para a variedade Icatu (c), em função das doses de silicato de cálcio $\left(\mathrm{CaSiO}_{3}\right)$ aplicadas ao substrato de plantio. cafeicultura brasileira, pois os solos são, de modo geral, pobres neste elemento (Reis Jr. \& Martinez, 2002).

Observaram-se diferenças significativas na EU de Fe e na EA de Mn pelas mudas de cafeeiro independente da variedade (Figura 3a,c), com interação significativa para a EA de Fe e para a ET de Mn para a variedade Icatu (Figura 3b,d). Com o aumento das doses de Si, observou-se redução da EU do Fe pelas mudas de cafeeiro até a dose $0,77 \mathrm{~g} \mathrm{dm}^{-3}$ de $\mathrm{CaSiO}_{3}$, com tendência de aumento da EU a partir daí (Figura 3a). Na variedade Icatu, a EA de Fe reduziu-se até a dose $0,40 \mathrm{~g} \mathrm{dm}^{-3}$ de $\mathrm{CaSiO}_{3}$, aumentando em seguida (Figura 3b).

Independentemente da variedade, a EA de Mn foi $13 \%$ maior com a dose $0,125 \mathrm{~g} \mathrm{dm}^{-3} \mathrm{de} \mathrm{CaSiO}_{3}$ do que na testemunha e $10 \%$ menor com a dose $0,5 \mathrm{~g} \mathrm{dm}^{-3}$ (Figura 3c). A ET de Mn na variedade Icatu reduziuse até a dose $0,63 \mathrm{~g} \mathrm{dm}^{-3}$ de $\mathrm{CaSiO}_{3}$ (Figura 3d), aumentando a seguir. Uma forma pela qual o Si pode proporcionar melhor equilíbrio nutricional em plantas de arroz diz respeito à sua capacidade de reduzir a absorção de Mn e Fe (Okuda \& Takahashi, 1964; Verma \& Minhas, 1987; Ma \& Takahashi, 1990). Quando plantas de arroz são adubadas com Si, ocorre aumento da oxidação do $\mathrm{Fe}^{2+}$ e do $\mathrm{Mn}^{2+}$ na superfície das raízes e, como resultado, esses nutrientes precipitam, não sendo absorvidos pela planta. Como existe forte afinidade entre $\mathrm{P}$ e $\mathrm{Fe}$ e $\mathrm{Mn}$, a relação entre esses elementos pode afetar a nutrição de $\mathrm{P}$. O $\mathrm{P}$ pode precipitar-se com o Fe na planta e combinarse com o Mn numa forma inativa.
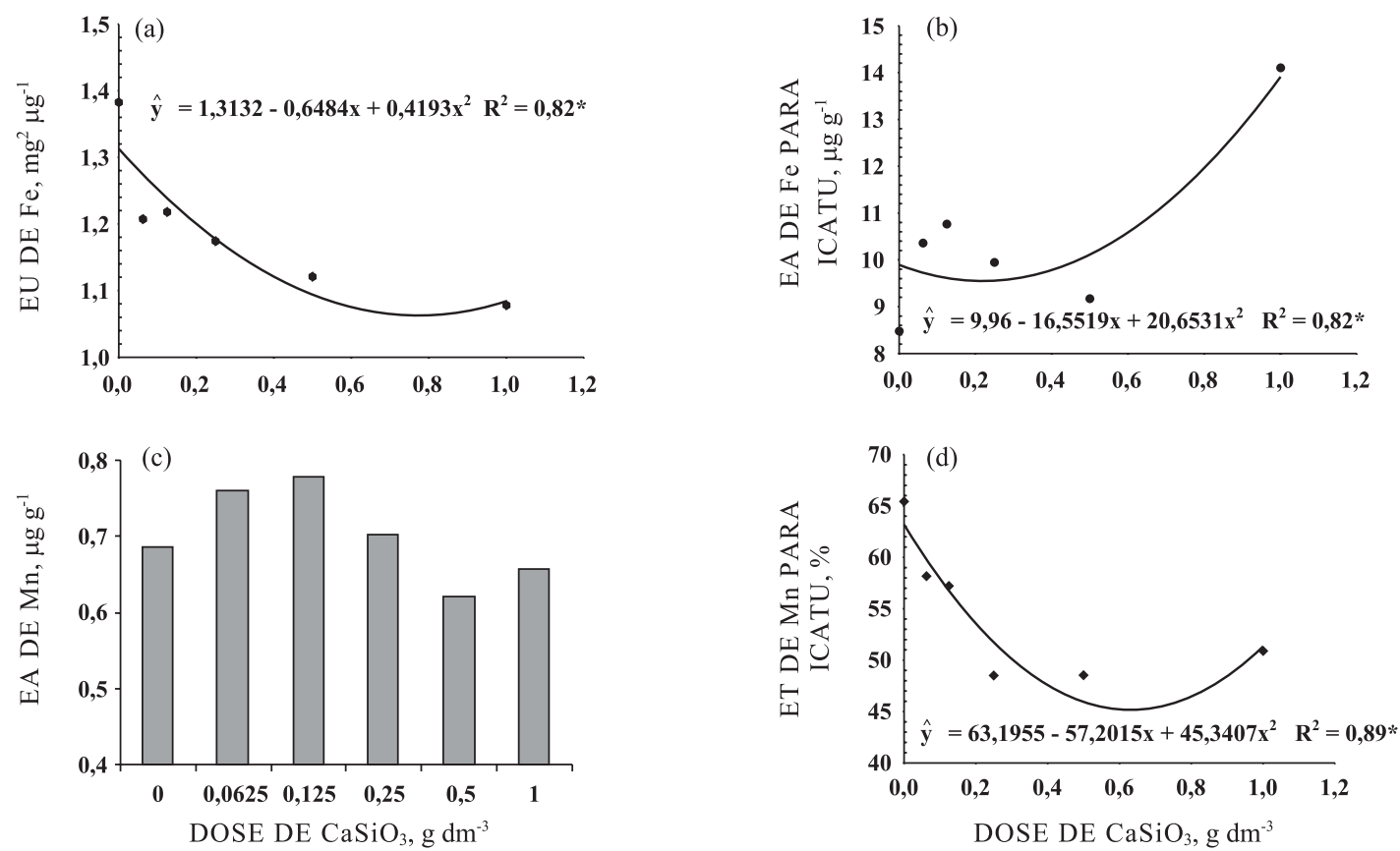

Figura 3. Eficiência de uso (EU) do ferro pelas mudas de cafeeiro independente da variedade (a); eficiência de absorção (EA) do ferro pela variedade Icatu (b); EA de Mn pelas mudas de cafeeiro (c) e eficiência de translocação (ET) de Mn pela variedade Icatu (d), em função das doses de silicato de cálcio $\left(\mathrm{CaSiO}_{3}\right)$ aplicadas no substrato de plantio. 
Os dados de EU do Si pelas mudas ajustaram-se à equação de regressão de forma quadrática e os valores diminuíram com o aumento das doses de silicato de Ca aplicados ao substrato de plantio. Essa redução ocorreu até $0,77 \mathrm{~g} \mathrm{dm}^{-3} \mathrm{de} \mathrm{CaSiO}_{3}$ (Figura 4a). Não houve ajuste de um modelo de regressão para explicar o fenômeno biológico da EA do Si pelas mudas, e a interação entre as variedades e as doses de $\mathrm{CaSiO}_{3}$ para a EA não diferiu significativamente.

Em trabalho com absorção e translocação de $\mathrm{Si}$ em mudas de eucalipto cultivadas em Latossolo e Cambissolo, Carvalho et al. (2003) indicaram um mecanismo de exclusão de Si para esta cultura, pois a EA aumentou enquanto a EU diminuiu. Os autores também observaram relação inversa entre o incremento das doses de Si no solo e a eficiência de uso do Si pela planta em Latossolo. Houve interação significativa entre ET e variedades. A resposta da ET às doses de Si ajustou-se aos modelos quadrático e linear. Para as variedades Catuaí e Icatu, observouse aumento linear da ET com o aumento das doses de
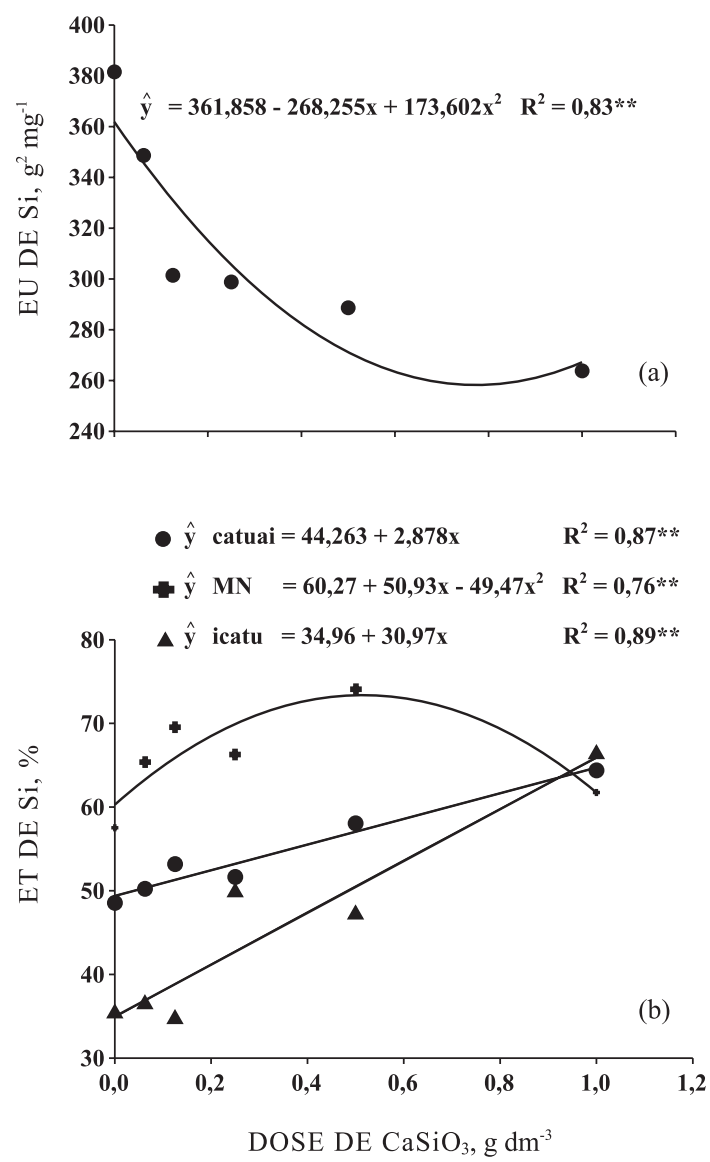

Figura 4. Eficiência de uso (EU) de Si pelas mudas de cafeeiro (a) e eficiência de translocação (ET) de Si nas mudas de cafeeiro das variedades Catuaí, Mundo Novo e Icatu (b), em função das doses de silicato de cálcio $\left(\mathrm{CaSiO}_{3}\right)$ aplicadas ano substrato de plantio. silicato. Para a Mundo Novo, a ET aumentou até a dose $0,52 \mathrm{~g} \mathrm{dm}^{-3} \mathrm{de} \mathrm{CaSiO}_{3}$ e reduziu sua eficiência a seguir (Figura 4b).

Na média, a variedade Mundo Novo translocou proporcionalmente mais Si para a parte aérea do que a Catuaí e a Icatu, isto é, para cada mg de Si absorvido, $0,66 \mathrm{mg}$ foi transportado para a parte aérea da Mundo Novo, enquanto para a Catuaí esse transporte foi de 0,54 mg e, para a Icatu, de 0,45 mg (Quadro 3). A adubação com Si pode aumentar a taxa fotossintética das plantas, pois o acúmulo de Si nas células da epiderme mantém as folhas mais eretas, aumentando a penetração da luz no dossel. O Si também diminui a transpiração excessiva, evitando ou reduzindo o estresse hídrico nas folhas e com isso estimula a fotossíntese e a atividade radicular. Dessa forma, o Si pode aumentar a absorção de água e, consequentemente, de nutrientes (Lima Filho, 2004).

\section{Relação do acúmulo do nutriente entre a parte aérea e as raízes}

Existem vários mecanismos relacionados às características morfológicas e fisiológicas da planta, os quais contribuem para o uso eficiente de nutrientes, dentre eles a alta relação entre a parte aérea e as raízes. Dessa forma, pode-se ter correlação direta para este atributo, ou seja, quanto maior a relação parte aérea/raízes, maior a eficiência nutricional da variedade (Fageria \& Baligar, 1993). Para a variedade Mundo Novo, a relação de acúmulo de N, P, K, Ca, B, Mn e Si entre parte aérea e raízes (PA/R) foi maior do que para as demais. Portanto, esta variedade foi mais eficiente na produção de matéria seca tanto da parte aérea quanto de raízes.

O crescimento radicular de Schizolobium parahyba var. amazonicum (Huber ex Ducke) Barneby, também conhecido como bandarra, essência florestal pertencente à família das LeguminosaeCesalpinoideae, foi afetado pela deficiência de nutrientes. O tratamento com omissão de $\mathrm{N}$ em solução nutritiva apresentou baixa relação PA/R, indicando menor produção de MS da parte aérea em relação à da raiz (Locatelli et al., 2007).

A relação $\mathrm{PA} / \mathrm{R}$ na variedade Catuaí foi maior para os nutrientes $\mathrm{Mg}, \mathrm{S}$ e $\mathrm{Cu}$, não diferindo da Icatu para o S. A variedade Icatu teve maior relação $P A / R$ para Zn e Fe (Quadro 4). Em mudas de eucalipto, pela análise da relação de acúmulo de Si da PA/R. Carvalho et al. (2003) observaram retenção do Si nas raízes, pois a relação $\mathrm{PA} / \mathrm{R}$ reduziu a partir de 60 dias após o transplantio, indicando redução da eficiência de translocação de Si pelo eucalipto com o avançar da idade das plantas. Nesse experimento, a variedade Mundo Novo apresentou maior relação PA/R corroborando com a afirmativa de que tem maior capacidade de produção de matéria seca, comparada à Catuaí e à Icatu. 
Quadro 4. Relação de acúmulo de nutrientes na parte aérea/raízes (PA/R) de variedades de cafeeiro cultivadas em tubetes independente dos tratamentos

\begin{tabular}{lcccc}
\hline Relação parte aérea/raízes (PA/R) & Catuaí & Mundo Novo & Icatu & CV (\%) \\
\hline Relação PA/R de N & $1,03 \mathrm{~b}$ & $1,26 \mathrm{a}$ & $1,04 \mathrm{~b}$ & 15,11 \\
Relação PA/R de P & $1,08 \mathrm{~b}$ & $1,60 \mathrm{a}$ & $1,13 \mathrm{~b}$ & 7,37 \\
Relação PA/R de K & $0,85 \mathrm{c}$ & $1,63 \mathrm{a}$ & $1,26 \mathrm{~b}$ & 17,84 \\
Relação PA/R de Ca & $0,99 \mathrm{~b}$ & $1,22 \mathrm{a}$ & $0,93 \mathrm{~b}$ & 13,60 \\
Relação PA/R de Mg & $1,11 \mathrm{a}$ & $0,54 \mathrm{c}$ & $0,94 \mathrm{~b}$ & 11,79 \\
Relação PA/R de S & $1,06 \mathrm{a}$ & $0,34 \mathrm{~b}$ & $1,05 \mathrm{a}$ & 17,67 \\
Relação PA/R de B & $1,06 \mathrm{~b}$ & $1,67 \mathrm{a}$ & $1,12 \mathrm{~b}$ & 13,56 \\
Relação PA/R de Cu & $1,48 \mathrm{a}$ & $0,25 \mathrm{c}$ & $1,10 \mathrm{~b}$ & 26,23 \\
Relação PA/R de Zn & $0,94 \mathrm{~b}$ & $0,13 \mathrm{c}$ & $1,06 \mathrm{a}$ & 20,50 \\
Relação PA/R de Fe & $0,91 \mathrm{~b}$ & $0,87 \mathrm{~b}$ & $1,57 \mathrm{a}$ & 23,97 \\
Relação PA/R de Mn & $1,09 \mathrm{~b}$ & $2,56 \mathrm{a}$ & $1,30 \mathrm{~b}$ & 25,41 \\
Relação PA/R de Si & $1,25 \mathrm{~b}$ & $2,21 \mathrm{a}$ & $0,91 \mathrm{c}$ & 35,40 \\
\hline
\end{tabular}

\section{CONCLUSÕES}

1. A variedade Icatu teve maior eficiência de absorção de $\mathrm{Mg}, \mathrm{S}, \mathrm{Cu}, \mathrm{Zn}, \mathrm{Fe}$ e $\mathrm{Si}$, maior eficiência de uso de N, K, Ca, B e Mn, não diferindo da Mundo Novo para N, Ke Mn e maior eficiência de translocação de N, S, Zn e de Fe. A Catuaí, por sua vez, absorveu mais os nutrientes $\mathrm{P}, \mathrm{K}, \mathrm{B}$ e Mn, não diferindo da Mundo Novo para $\mathrm{P}, \mathrm{K}$ e Mn; teve também maior eficiência de uso de $\mathrm{Mg}, \mathrm{S}, \mathrm{Cu}, \mathrm{Zn}, \mathrm{Fe}$ e $\mathrm{Si}$, provavelmente devido à melhor eficiência de translocação desses nutrientes, exceto para o Fe e o Si. Entretanto, a Mundo Novo foi a variedade com maior peso da parte aérea e total. Além de ter sido a mais eficiente na absorção de N, P, K, Ca, Mg, Mn e $\mathrm{Si}$, teve maior eficiência de uso de $\mathrm{N}, \mathrm{P}, \mathrm{K}$ e Mn e maior translocação de $\mathrm{P}, \mathrm{K}, \mathrm{Ca}, \mathrm{B}, \mathrm{Mn}$ e $\mathrm{Si}$, sendo, por isso, considerada a mais eficiente nutricionalmente.

2. A aplicação de silicato alterou a eficiência nutricional das variedades de cafeeiro, principalmente das variedades Icatu e Mundo Novo, e praticamente não alterou a eficiência nutricional da Catuaí.

\section{LITERATURA CITADA}

ABICHEQUER, A.D. \& BOHNEN, H. Eficiência de absorção, translocação e uso de fósforo por variedades de trigo. R. Bras. Ci. Solo, 22:21-26, 1998.

ANGHINONI, I.; VOLKART, C.R.; FATTORE, N. \& ERNANI, P.R. Morfologia de raízes e cinética da absorção de nutrientes em diversas espécies e genótipos de plantas. R. Bras. Ci. Solo, 13:355-361, 1989.
DUNCAN, R.R. \& BALIGAR, V.C. Genetics and physiological basis of nutrient uptake and use efficiency. In: BALIGAR, V.C. \& DUNCAN, R. R., eds. Crops as enhancers of nutrient use. New York, Academic Press, 1990. p.335 .

CARVALHO, R.; FURTINI NETO, A.E.; CURI, N. \& RESENDE, A.V. Absorção e translocação de silício em mudas de eucalipto cultivadas em Latossolo e Cambissolo. Ci. Agrotec., 27:491-500, 2003.

CORRÊA, J.B.; GARCIA, A.W.R. \& COSTA, P.C. Extração de nutrientes pelos cafeeiros Mundo Novo e Catuaí. In: CONGRESSO BRASILEIRO DE PESQUISAS CAFEEIRAS, 10., Poços de Caldas, 1983. Anais. Rio de Janeiro, IBC/GERCA, 1983. p.117-183.

FAGERIA, N.K. Otimização da eficiência nutricional na produção das culturas. R. Bras. Eng. Agric. Amb., 2:6-16, 1998.

FAGERIA, N.K. \& BALIGAR, V.C. Screening crop genotypes for mineral stresses. In: WORKSHOP ON ADAPTATION OF PLANTS TO SOIL STRESSES, 1993, Lincoln. Proceedings. Lincoln, University of Nebraska, 1993. p.142159.

FURLANI, A.M.C.; BATAGLIA, O.C. \& AZZINI, L.E. Comportamento diferencial de linhagens de arroz na absorção e utilização de nitrogênio em solução nutritiva. R. Bras. Ci. Solo, 10:51-59, 1986.

GALLO, J.R. \& FURLANI, P.R. Determinação de silício em material vegetal pelo método colorimétrico do azul de molibdênio. Bragantia, 37:5-11, 1978.

GERLOFF, G.C. \& GABELMAN, W.H. Genetic basis of inorganic plant nutrition. In: LÄUCHLI, A. \& BIELESKI, R.L., eds. Inorganic plant nutrition. Berlin, SpringerVerlag, 1983. p.453-480. (Encyclopedia of Plant Physiology, v. $15 \mathrm{~B})$ 
KORNDÖRFER, G.H. \& GASCHO, G.J. Avaliação de fontes de silício para o arroz. In: CONGRESSO NACIONAL DE ARROZ IRRIGADO, 1., Pelotas, 1999. Anais. Pelotas, 1999. p.313-316.

KORNDÖRFER, G.H. Elementos benéficos In: FERNANDES, M.S., ed. Nutrição mineral de plantas. Viçosa, MG, Sociedade Brasileira de Ciencia do Solo, 2006. p.355-374.

LI, B.; MCKEAND, S.E. \& ALLEN, H.L. Genetic variation in nitrogen use efficiency of loblolly pine seedlings. For. Sci., 37:613-626, 1991.

LIMA FILHO, O.F. O silício: Desenvolvimento da agricultura. Disponível em: <http://www.plantearural.com.br/ Artigos.asp?ID=75>. Acesso em 30 ago. 2004.

LOCATELLI, M.; MELO, A.S.; LIMA, L.M.L \& VIEIRA, A.H. Deficiências nutricionais em mudas de Schizolobium parahyba var. amazonicum. R. Bras. Bioci., 5:648-650, 2007.

MA, J. \& TAKAHASHI, E. Effect of silicon on the growth and phosphorus uptake of rice. Plant Soil, 126:115-119, 1990.

MALAVOLTA, E.; VITTI, G.C. \& OLIVEIRA, S.A. Avaliação do estado nutricional das plantas: Princípios e aplicações. 2.ed. Piracicaba, POTAFOS, 1997.

MALAVOLTA, E. Manual de nutrição mineral de plantas. Piracicaba, Ceres, 2006. 638p.

MARTINEZ, H.E.P.; NOVAIS, R.F.; SACRAMENTO, L.V.S. \& RODRIGUES, L.A. Comportamento de variedades de soja cultivadas sob diferentes níveis de fósforo: II. Translocação do fósforo absorvido e eficiência nutricional. R. Bras. Ci. Solo, 17:239-244, 1993.

MATIELLO, J.B.; SANTINATO, R.; GARCIA, A.W.R.; ALMEIDA, S.R. \& FERNÁNDEZ, D.R. Cultura do café no Brasil - novo manual de recomendações. Rio de Janeiro, MAPA/PROCAFÉ, 2002. 386p.

MÉNDÉZ BALDEON, J.R. Efeito da ação alcalinizante e da competição entre silicato e fosfato na eficiência do termofosfato magnesiano em solos ácidos. Piracicaba, Escola Superior de Agricultura Luiz de Queiroz, 1995. 88 p. (Tese de Doutorado)

OKUDA, A. \& TAKAHASHI, E. The role of silicon. In: HOPKINSIB, J., ed. The mineral nutrition of the rice plant. In: SYMPOSIUM AT THE INTERNATIONAL RICE RESEARCH INSTITUTE, São Paulo, 1964. Proceedings. São Paulo, Kosmos, 1964. p.123-146.
REIS JR., R.A. \& MARTINEZ, H.E.P. Adição de Zn e absorção, translocação e utilização de Zn e $\mathrm{P}$ por variedades de cafeeiro. Sci. Agric., 59:537-542, 2002.

SANDS, R. \& MULLIGAN, D.R. Water and nutrient dynamics and tree growth. For. Ecol. Manag., 30:91-111, 1990.

SANTOS BOTELHO, D.M.; POZZA, E.A.; POZZA, A.A.A.; CARVALHO, J.G.; BOTELHO, C.E. \& SOUZA, P.E. Intensidade da cercosporiose em mudas de cafeeiro em função de fontes e doses de silício. R. Fitopatol. Bras., 30:582-588, 2005 .

SIDDIQI, M.Y. \& GLASS, A.D.M. Utilization index: A modified approach to the estimation and comparison of nutrient utilization efficiency in plants. J. Plant Nutr., 4:289-302, 1981.

SOUZA, M.E. Correlação adulto juvenil para eficiência nutricional e comportamento de clones de Eucalyptus grandis em dois níveis de fertilidade do solo. Viçosa, MG, Universidade Federal de Viçosa, 1994. 102p. (Tese de Mestrado)

SOUZA, R.B. Níveis críticos de enxofre em solos e em folhas de cultivares de café. Viçosa, MG, Universidade Federal de Viçosa, 1999. 88p. (Tese de Doutorado)

SUNARPI ANDERSON, J.W. Effect of sulfur nutrition on the redistribution of sulfur in vegetative soybean plants. Plant Physiol., 112:623-631, 1996.

SWIADER, J.M.; CHYAN, Y. \& FREIJI, F.G. Genotypic differences in nitrate uptake and utilization efficiency in pumpkin hybrids. J. Plant Nutr., 17:1687-1699, 1994.

TOMAZ, M.A.; SILVA, S.R.; SAKIYAMA, N.S. \& MARTINEZ, H.E.P. Eficiência de absorção, translocação e uso de cálcio, magnésio e enxofre por mudas enxertadas de Coffea arabica. R. Bras. Ci. Solo, 27:885-892, 2003.

VERMA, T.S. \& MINHAS, R.S. Effect of iron and manganese interaction on paddy yield and iron and manganese nutrition in silicon-treated and untreated soils. Soil Sci., 147:107-115, 1987.

WHITEAKER, G.; GERLOFF, G.C.; GABELMAN, W.H. \& LINDGREN, D. Intraspecific differences in growth of beans at stress levels of phosphorus. J. Am. Soc. Hortic. Sci., 101:472-475, 1976.

WUEST, S.B. \& CASSMAN, K.G. Fertilizer-nitrogen use efficiency of irrigated wheat. I. Uptake efficiency of preplant versus late-season application. Agron. J., 84:682688, 1992 